\title{
Syntactic structure and natural language mediation $^{1,2,3}$
}

ALEXANDER J. WEARING, Yale University, New Haven, Conn. 06510, CLINTON B. WALKFR, University of Rochester, Rochester, N.Y. 14627, and WILLIAM E. MONTAGUE, University of Illinois, Urbana, Ill. 61801

If natural language is important in the memory devices that Ss generate in verbal-learning experiments, it should follow that a memory device's effectiveness is related to its grammatical structure. Data are reported that show only a weak relationship between the grammatical characteristics of reported mediational devices and acquisition in a paired-associate learning task.

Several years of research on the memory devices that $S$ s report using in verbal learning experiments have established that these devices are closely correlated with $\mathrm{Ss}^{\prime}$ performance (for example, Adams, 1967 Delin, 1969; Montague \& Wearing, 1967a,b; Wearing, Walker, \& Montague, 1967). It has been tacitly assumed that these reported memory devices are based on natural language, and this assumption is reflected in their being labeled "natural language mediators" (NLMs) by Adams and Montague (Adams, 1967; Montague, Adams, \& Kiess, 1966; Montague \& Kiess, 1968). Although these memory devices are reported by means of language, the medium of the report may or may not reflect the nature of what has been reported, which could well be a sense impression or some other nonverbal process.

Under one influential theory of language (Chomsky, 1965), the surface structure of a phrase or sentence provides one access to its underlying structure, and that underlying structure provides the information necessary for its meaningful interpretation. If natural language is of crucial importance to an NLM, it should follow that an NLM's effectiveness as a mediator is related to its grammatical structure. The ease with which a learned item can be retrieved should be related to its syntactic function in the relevant NLM. Moreover, if mediators derive mainly from the natural language, then it should make little difference to a S's performance whether he is instructed to link items by means of grammatical sentences or is given no directions about grammatical form, being told only to create a memory device for the cue pair.
To investigate the role of grammatical rules in so-called natural language mediation, an experiment was run in which Ss learned and recalled a list of CVC English word pairs under two different sets of learning instructions. One group of Ss was told to write a sentence with the pair of items, and a second group was told to write out a memory device for the pair, if such a device could be generated. The grammatical structure of these sentences and memory devices were then analyzed. SUBJECTS

Twelve fernale undergraduates from the University of Illinois comprised the Ss.

\section{MATERIALS}

Fifteen pairs of CVCs were used. Twenty-nine of the items were English words and one (TEL) was a near word. These CVC pairs ranged from easy (VET-FOX, PEW-NUN, etc.) to hard (DUG-ZIP, BUN-TOG, etc.). These 15 pairs were embedded in a list of 80 pairs consisting mainly of CVC English words. PROCEDURE

An equal number of $\mathrm{Ss}$ was assigned to two experimental groups. These Ss were run on the University of Illinois PLATO system (Bitzer, Hicks, Johnson, \& Lyman, 1967). Each $S$ sat at a booth containing both a television display for showing instructions and stimuli and a typewriter-like keyset for responding.

Following some instructions, Ss were shown each stimulus pair for a period of $15 \mathrm{sec}$. The two experimental groups were distinguished by the response they were told to make during that period. The first (sentence) group was told that if they could form a sentence with the pair being shown, to write it out. The second (NLM) group was told to write out any memory device they could generate for the pair. In order to equate written repetitions of the pairs, all Ss were told that if they failed to generate a sentence of an NLM, to write down the two stimulus words.

Following the presentation of the list, a paced recall trial was given in which $S$ s had to type the right-hand member of each pair during the $9 \mathrm{sec}$ that the left-hand member was shown on the screen. The letters typed by the Ss were displayed on the right of the stimulus until the next stimulus was presented.

\section{RESULTS}

Mediators were written down for 74 of the 90 items in the NLM group and 66 of the 90 items in the sentences group Although the mediators for the NLM group were not required to be grammatical sentences, they always did comprise grammatical or near grammatical word strings, e.g., "FAN and SIP in the sun," albeit incomplete as sentences.

A number of hypotheses concerning the role of grammatical rules in natural language mediation are stated and examined in turn by chi-square tests.

First, it seems plausible that the parts of a sentence easiest to recall are the nuclear words, the subject, verb, and object (if the verb is transitive) or the subject, verb, and predicate adjective or predicate nominalization (if the verb is intransitive). Hence, the recall of a pair should vary directly with the number of items in the pair that are nuclear parts.

There were no differences due to the sentence and the NLM instructions, and the percentages of pairs correct for 0,1 , and 2 items as nuclear words in the surface structure were $41 \%(7 / 17), 44 \%(22 / 50)$, and $49 \%(36 / 73)$, respectively. The trend, though in the predicted direction, is insignificant.

A second plausible hypothesis is that if the two items of a pair are in the same underlying sentence, then they are more likely to be contained in the same mediational chunk, hence will be recalled better than items that are not in the same deep sentence. In fact, there were no differences between the sentence and NLM conditions (where NLMs not forming a complete sentence were omitted) and although the items stored in the same deep sentence were recalled better $[51 \%(47 / 92)$ to $42 \%(18 / 43)]$, again the difference was not significant.

A third hypothesis is that if the mediator joining two items links them by the conjunction and, then recall should be poorer than if they were linked by either a verb or a preposition (Rohwer, 1966). Verbs and prepositions imply some "semantic interaction" between the items that they link, hence resulting in a more intimate meaning relation than occurs with conjunctions, which provide a semantically parallel relationship. Our results did not support this hypothesis, $61 \%(14 / 23)$ of the items conjoined by and and $45 \%$ (53/117) of the items conjoined by a verb or a preposition being recalled correctly $(\mathrm{p}<.20)$.

Fourthly, it might be expected that the presence of a surface subject in the mediator will expedite recall if one of the items was that subject. There was no statistically reliable support for such an expectation $(\mathrm{p}<.20)$, although $58 \%$ (26/45) of the pairs for which one of the items was subject of the mediator was recalled, as against $46 \%(27 / 59)$ that did not have one of the items as the subject of 
the mediator ( 36 cases did not have a surface subject).

The fifth hypothesis was that items would be recalled better if they were incorporated as whole lexical items into the sentence or NLM than if they were embedded in other words. According to this prediction, BUN-TOG should be recalled better when the memory device was "She wore a BUN on her TOGs" than when it was "The BUNny worked the TOGgle switch." An additional transformation of information, in part semantic and phonetic, is necessary when the item is embedded in a different word. If the transformation is not recalled, then correct recall of the memory device is useless. There was some support for this proposition, in that $56 \%(55 / 99)$ of the items that retained their integrity were correctly recalled as opposed to $30 \%$ (12/44) of the items that were combined into larger words $(\mathrm{p}<.01)$.

\section{DISCUSSION}

Although the results confirmed those of other investigators in that items for which a mediator was reported were recalled significantly better than other items ( $55 \%$ to $14 \%$, respectively), linguistic structure did not have a consistent or strong influence on recall. No differences were obtained between the sentence and the NLM groups, which indicates that the instructions exerted no differential constraining effects upon the Ss. However, the inconsistency with which grammatical form relates to the effectiveness of a mediator casts doubt upon the primacy of linguistic variables in the formation of memory devices. It follows that the label of NLM is something of a misnomer unless it is intended to refer only to the means by which a memory device is communicated to an observer.

Several explanations can be advanced for the failure of our predictions. First, perhaps the wrong linguistic relations were examined. This is quite possible, but it would be hard to find linguistic variables that are now considered more basic or more general. Second, the task or the verbal materials may be so artificial that they do not readily expose linguistic organization and processes in learning. It is quite possible that a learning task that was devised for studying S-R associations may not be suitable for studying the use of rules and cognitive structures. Third, it is by no means certain that the mediators that the Ss wrote down were, in fact, the mediators that they used-the reported memory devices may have possibly been generated in response to the task demands of the experiment and not utilized in learning the items.

However, because four of five results that we examined were in the predicted direction, we cannot discard the possibility that grammatical structures play a role in the elaboration of memory devices, albeit not a primary one. Having raised the issue of the role of syntactic structure in natural language mediation, we find it prudent to leave the question open.

\section{REFERENCES}

ADAMS, J. A. Human memory. New York: McGraw-Hill, 1967.

BITZER, D. L., HICKS, B., JOHNSON, R., \& LYMAN, E. R. The PLATO system: Current research and development. IEEE Transactions on Human Factors in Electronics, 1967, 8, 64-70.

DELIN, P. S. Learning and retention of English words with successive approximations to a complex mnemonic instruction. Psychonomic Science, 1969, 17, 87-89.

CHOMSKY, A. N. Aspects of a theory of syntax. Cambridge, Mass: M.I.T. Press, 1965.

MONTAGUE, W. E., ADAMS, J. A., \& KIESS, H. $O$. Forgetting and natural language mediation. Journal of Experimental Psychology, 1966, 72, 829-833.

MONTAGUE, W. E., \& KIESS, H. O. The associability of CVC pairs. Joumal of Experimental Psychology Monograph, 1968, 78, No. 2, Part 2.

MONTAGUE, W. E., \& WEARING, A. J. The complexity of natural language mediators and its relation to learning. Psychonomic Science, 1967a, 7, 135-136.

MONTAGUE, W. E., \& WEARING, A. J. Natural language mediation: $A$ source of interference with extra-experimental interference. Psychonomic Science, 1967b, 9, 317-318.

ROHWER, W. D. Constraint, syntax, and meaning in paired-associate learning. Journal of Verbal Learning \& Verbal Behavior, 1966, 5, 541-547.

WEARING, A. J., WALKER, K. B., \& MONTAGUE, W. E. Recall of paired associates as a function of their associability. Psychonomic Science, 1967, 9, 533-534. NOTES

1. This research was supported in part by the Joint Services Electronic Program (U.S. Army, U.S. Navy, and U.S. Air Force) under Contract No. DA 28043 AMC 00073(E); in part by ONR Nonr-3985(08); and in part by the Computer-based Educational Research Laboratory at the University of Illinois.

2. The authors are grateful to Rosemary Wearing, Linda Kalmanek, Marcia Harms, Valerie Kussler, Pamela Polson, Jillian Rewortz, and Barbara Larick for their assistance in the collection and analysis of the data presented here.

3. Please address all requests for reprints to Alexander J. Wearing, Yale University, Department of Psychology, 333 Cedar St., New Haven, Conn. 06510.

\section{A test of the spew hypothesis using intralist repetition and a free-recall task ${ }^{1}$}

\section{SHARRON S. WIKE and EDWARD L. WIKE, University of Kansas, Lawrence, Kans. 66044}

The role of intralist repetition on free recall was investigated using 43 college students. In each of four lists, 5 words were repeated three times each and 15 words were given once. The same 20 words were used for all four lists, but the words that were repeated varied across lists. It was found that during output the repeated words had significantly higher mean position than did the nonrepeated words. The result agreed with the spew hypothesis of Underwood \& Schulz, (1960).

According to the spew hypothesis of Underwood \& Schulz (1960), the order of output of verbal items is related to the frequency of input. It is assumed that more frequently experienced verbal items are more available and are recalled sooner than less frequently experienced items.

Underwood and Schulz found support for this hypothesis in a number of studies (e.g., Brown, 1915; Cromwell, 1956; Johnson, 1956; Howes, 1957) in which the frequency of occurrence was established outside the laboratory. In Experiment 1 (Underwood and Schulz), frequency was manipulated experimentally and output was measured in free recall. The $S$ was given familiarization training on 40 low-association-value nonsense syllables. The $\mathrm{S}$ was exposed to a given syllable 40 , 20,10 , or 1 time and was instructed to spell the syllable upon each exposure. At different intervals during the familiarization training, $S$ had five test 